\title{
Signal Enhancement by a Multi-layered Substrate for Mutagen Detection Using an SOS Response-induced Green Fluorescent Protein in Genetically Modified Escherichia coli
}

\author{
Hiroki Eтон, Mitsuru YASUdA, and Takuo Акімото ${ }^{\dagger}$ \\ School of Bioscience and Biotechnology, Tokyo University of Technology, 1404-1 Katakura, Hachiouji, \\ Tokyo 192-0982, Japan
}

\begin{abstract}
In this paper, we describe a method to enhance the fluorescence signal of mutagen detection using SOS response-induced green fluorescence protein (GFP) in genetically modified Escherichia coli using a multi-layered substrate. To generate E. coli that express SOS response-induced GFP, we constructed a plasmid carrying the RecA promoter located upstream of the GFP gene and used it to transform E. coli BL21. The transformed strain was incubated with mitomycin C (MMC), a typical mutagen, and then immobilized on a multi-layered substrate with $\mathrm{Ag}$ and a thin $\mathrm{Al}_{2} \mathrm{O}_{3}$ layer on a glass slide. Since the multi-layered substrate technique is an optical technique with potential to enhance the fluorescence of fluorophore placed on top of the substrate, the multi-layered substrate was expected to improve the fluorescence signal of mutagen detection. We obtained an average 14-fold fluorescence enhancement of MMC-induced GFP in the concentration range 1 to $1000 \mathrm{ng} / \mathrm{ml}$. In addition, the lower detection limit of MMC was improved using this technique, and was estimated to be $1 \mathrm{ng} / \mathrm{ml}$ because of an enlargement of the difference between the blank and the signal of $1 \mathrm{ng} / \mathrm{ml} \mathrm{of} \mathrm{MMC}$.
\end{abstract}

(Received August 30, 2011; Accepted October 27, 2011; Published December 10, 2011)

\section{Introduction}

Mutagens, such as benzopiren, mitomycin C (MMC), and others, are chemicals found in the environment and food that have the potential to induce genotoxic and carcinogenic effects. Generally modified Salmonella typhimurium, which requires histidine for growth, has been widely employed in the Ames test to detect mutagens. ${ }^{1-3}$ In this test, $S$. typhimurium is incubated with a mutagen, and the number of $S$. typhimurium colonies that appears is counted. The lower detection limit of this test has been reported to be $70 \mathrm{pg} / \mathrm{ml}$ for MMC. ${ }^{3,4}$ However, it requires $48 \mathrm{~h}$ of incubation.

For the rapid detection of mutagens, new techniques have been developed using bacterial SOS response, which is an inherent response to mutagen-induced DNA damage. ${ }^{5-8}$ The $\operatorname{RecA}$ promoter located upstream of the $\operatorname{RecA}$ gene plays an important role in the SOS response. In the absence of DNA damage, the LexA protein suppresses $\operatorname{Rec} A$ gene expression by binding to a specific sequence of the RecA promoter. However, when DNA is damaged by mutagens, the RecA gene is expressed because the LexA protein is self-cleaved, and hence released from the RecA promoter. ${ }^{9-12}$ Therefore, if a fusion gene carrying a green fluorescence protein (GFP) gene located downstream of the RecA promoter could be constructed and used to transform Escherichia coli BL21, mutagen-induced DNA damage could be detected on the expression of GFP in this strain. This SOS response-based mutagen assay reportedly requires an incubation of approximately $2 \mathrm{~h} .{ }^{13,14}$ However, its lower detection limit has

† To whom correspondence should be addressed.

E-mail: akimoto@bs.teu.ac.jp been reported to be $3 \mathrm{ng} / \mathrm{ml}$ for MMC, which is about 40-times lower than that of the Ames test. ${ }^{4,14}$

A multi-layered substrate fabricated with a metal and a dielectric thin layer has been known to enhance the fluorescence signal. When rhodamine B was placed on top of a multi-layered substrate with about $1 \mu \mathrm{m}$ thick $\mathrm{Ag}$, and a subsequent about $90 \mathrm{~nm}$ thick $\mathrm{LiF}$ or $\mathrm{Al}_{2} \mathrm{O}_{3}$ layer on a glass slide (Fig. 1),

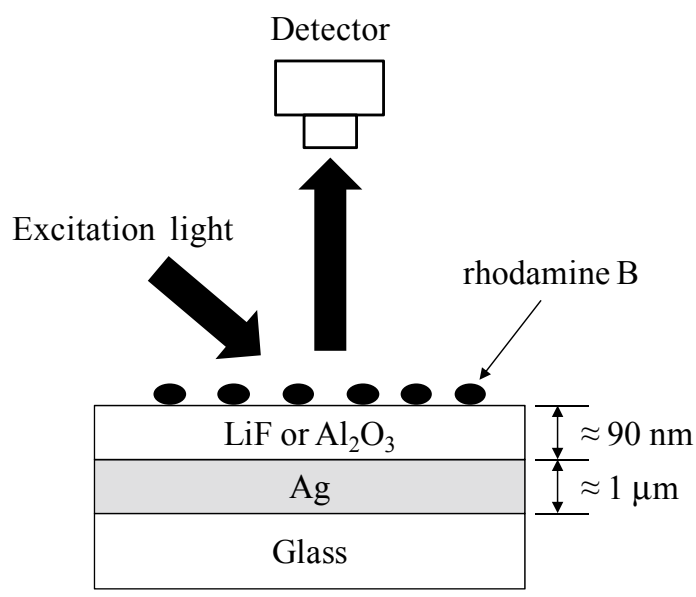

Fig. 1 Schema of the multi-layered substrate. The fluorescence enhancement is accounted for the interference of excitation and emission light in the dielectric layer of the multi-layered substrate. The thicknesses of the $\mathrm{Ag}$ and the dielectric layer were approximately $1 \mu \mathrm{m}$ and $90 \mathrm{~nm}$ for the maximum fluorescence enhancement of rhodamine B placed on top of the dielectric. 
Table 1 Sequences of RecA promoter and PCR primers

RecA promoter ${ }^{\text {a }} 5^{\prime}$-atttctacaaacacttgatactgtatatatatacagtataa ttgcttcaacagaacatattgactatccggtattacceggcatgac- $3^{\prime}$ Forward primer $5^{\prime}$-agcttt agatctatt tctacaaaacacttgatac- $3^{\prime}$ Reverse primer $5^{\prime}$-acttaatctagagtcatgccgggtaatac-3'

a. The sequence of LexA binding site (italic) was decided according with Refs. 21 and 22.

the fluorescence intensity of rhodamine B could be enhanced by about 100-fold compared with a bare glass substrate. ${ }^{15-18}$ The fluorescence enhancement could account for the interference of both excitation and emission light in the $\mathrm{Al}_{2} \mathrm{O}_{3}$ layer. According to previous reports, a high-contrast image of a cell stained with a fluorophore was successfully obtained using the multilayered substrate. ${ }^{19,20}$ Based on these reports, we speculated that the multi-layered substrate enhances the fluorescence signal of the SOS response-based mutagen assay, and hence leads to an improvement of the detection limit.

In this paper, we describe mutagen detection using genetically modified $E$. coli immobilized on a multi-layered substrate. We constructed a plasmid carrying the GFP gene located downstream of the $\operatorname{Rec} A$ promoter, and used it to transform E. coli BL21. To confirm that GFP expression was mutagen induced, the transformed E. coli strain was cultured in the presence of mutagens, and analyzed for GFP expression. Finally, E. coli that expressed the GFP was immobilized on a multi-layered substrate, and the enhancement of the fluorescence signal and the detection limit by the SOS response-induced GFP was quantitatively estimated.

\section{Experimental}

\section{Reagent and materials}

MMC and $N$-methyl- $N$-nitro- $N$-nitrosoguanidine (MNNG) used as mutagens were purchased from Wako. Ampicillin used for selection of transformed cell was purchased from Wako. Isopropyl $\beta$-D-1-thiogalactopyranoside (IPTG) used for T7 promoter-induced GFP expression was purchased from Wako. Luria-Bertani (LB) medium was prepared with $10 \mathrm{~g} / \mathrm{l}$ of trypton (Bact), $5 \mathrm{~g} / \mathrm{l}$ of yeast extract (Bact) and $10 \mathrm{~g} / \mathrm{l}$ of $\mathrm{NaCl}$ (Wako) 3-Aminopropyltrimethoxysilane (APTS) used to modify the substrates was purchased from Sigma. The RecA promoter oligonucleotide and primers for PCR amplification were synthesized by Tsukuba Oligo Services.

\section{Plasmid construction and E. coli transformation}

The RecA promoter was amplified by PCR using oligonucleotide primers incorporating the cleavage site for $B g l \mathrm{II}$ and $\mathrm{Xba}$ I restriction endonuclease. The sequences of the $R e c A$ promoter and the primers used for PCR amplification are provided in Table 1.

After PCR amplification of the RecA promoter, the PCR fragment was analyzed by gel electrophoresis, and confirmed to be approximately $120 \mathrm{bp}$ (data not shown). A plasmid, pQBIT7-GFP, (Qbiogene) carrying a T7 promoter located upstream of the GFP gene was used to insert the RecA promoter Before inserting the $R e c A$ promoter, the $\mathrm{T} 7$ promoter was removed from pQBIT7-GFP using $B g l$ II and Xba I. The PCR fragment of the $R e c A$ promoter was digested with $B g l$ II and $X b a \mathrm{I}$, and subsequently ligated into pQBIT7-GFP between the $B g l$ II and Xba I sites. The constructed plasmid carrying the
RecA promoter was designated $\mathrm{pQBIrecA-GFP.}$

E. coli BL21 was transformed with plasmid pQBIrecA-GFP by using a heat shock protocol. The transformed strain was selected on an LB agar plate containing $100 \mu \mathrm{g} / \mathrm{ml}$ ampicillin. The selected strains were analyzed with colony PCR to confirm the presence of the correctly sized insert, and were stored at $-80^{\circ} \mathrm{C}$ in $60 \%$ glycerol.

\section{SOS response-based mutagens assay}

E. coli BL21 transformed with pQBIrecA-GFP was inoculated in the $\mathrm{LB}$ medium containing $100 \mu \mathrm{g} / \mathrm{ml}$ ampicillin at $37^{\circ} \mathrm{C}$ for $12 \mathrm{~h}$ as a pre-incubation procedure. The cell suspension was then inoculated into a fresh LB medium containing $100 \mu \mathrm{g} / \mathrm{ml}$ ampicillin, and incubated until $\mathrm{OD}_{660}$ was 0.5 (log phase of growth). MMC or MNNG at the desired concentration was added to the LB medium, and the cells were further incubated at $26^{\circ} \mathrm{C}$. To confirm the fluorescence of the SOS response-induced GFP, the cell suspension was pipetted into microtiter plates, and the fluorescence was measured using a microplate reader (Tecan).

\section{Fabrication of a multi-layered substrate}

A microscope glass slide was washed with ethanol by ultrasonication. After drying the cleaned slide, we deposited $\mathrm{Cr}$ as an adhesive layer for $\mathrm{Ag}, \mathrm{Ag}$ as a metal layer, and $\mathrm{Al}_{2} \mathrm{O}_{3}$ as a dielectric layer on the slide using sputtering deposition (CFS-4ES, Shibaura). The thicknesses of the $\mathrm{Cr}, \mathrm{Ag}$ and $\mathrm{Al}_{2} \mathrm{O}_{3}$ layers were controlled by time for the sputtering deposition, and determined to be 5,500 and $0-200 \mathrm{~nm}$, respectively, using a surface profilemeter (DekTak, Ulvac).

\section{Immobilization of E. coli on a multi-layered substrate}

To enhance efficiently the fluorescence by a multi-layered substrate, the fluorophore should be placed on top of the surface. Hence, we attempted to immobilize the cell that express GFP on the multi-layered substrate. To immobilize E. coli BL21 on the multilayered substrate, the surfaces of the substrate were modified with an amino group. For this, the substrate was immersed for $1 \mathrm{~h}$ in $1 \%$ APTS dissolved in ultra-pure water for $1 \mathrm{~h}$, followed by rinsing with ultra-pure water, and dried at 110 degrees for $1 \mathrm{~h}$.

The $E$. coli cell suspension in LB medium was centrifuged at $3000 \mathrm{rpm}$ for $3 \mathrm{~min}$, and the medium was replaced with $0.067 \mathrm{M}$ phosphate buffer (PB, $\mathrm{pH}$ 7.4). Following this, $0.125 \%$ glutaraldehyde in $\mathrm{PB}$ was mixed with an equal volume of the cell suspension in $\mathrm{PB}$, and $1 \mu \mathrm{l}$ of the mixture was spotted onto the multi-layered substrate to immobilize the cells. With this procedure, the amino group on the cell membrane was cross-linked to the amino group of the modified substrate, and thereby the cell could be immobilized on the substrate. For a comparison, the same immobilization procedure was performed for a bare glass substrate. The fluorescence of the immobilized E. coli was measured using a fluorescence imager (Typhoon, $\mathrm{GE})$.

\section{Results and Discussion}

\section{Optimization of the $\mathrm{Al}_{2} \mathrm{O}_{3}$ layer thickness}

The $\mathrm{Al}_{2} \mathrm{O}_{3}$ layer thickness of the multi-layered substrate was optimized to obtain the maximum fluorescence enhancement. To accomplish this, E. coli expressing GFP induced by the T7 promoter with $1 \mathrm{mM}$ IPTG, which was independent of the SOS response, was immobilized on the multi-layered substrate and the bare glass substrate; the fluorescence of GFP was then 


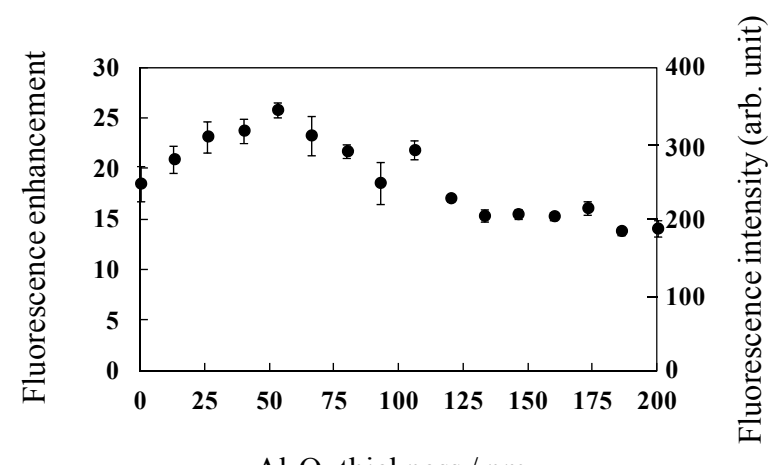

Fig. 2 Dependence of the fluorescence enhancement on the $\mathrm{Al}_{2} \mathrm{O}_{3}$ layer thickness. The E. coli that expressed GFP by the T7 promoter was immobilized on a multi-layered substrate and a bare glass substrate. The fluorescence enhancement was determined as the fluorescence using the multi-layered substrate divided by that using the bare glass substrate; $n=4$.

measured. The fluorescence enhancement was determined as the fluorescence using the multi-layered substrate divided by the fluorescence using the bare glass substrate (Fig. 2).

The fluorescence enhancement changed with the $\mathrm{Al}_{2} \mathrm{O}_{3}$ thickness; a maximum of 25-fold fluorescence enhancement was achieved with a 50-nm thick $\mathrm{Al}_{2} \mathrm{O}_{3}$ layer. However, the fluorescence enhancement of GFP using the multi-layered substrate should have changed periodically with the $\mathrm{Al}_{2} \mathrm{O}_{3}$ layer thickness, providing the maximum of 100 -fold enhancement. The low-fluorescence enhancement of GFP was due to the large size of $E$. coli compared to the $\mathrm{Al}_{2} \mathrm{O}_{3}$ layer thickness. Since the fluorescence enhancement was mainly attributed to the interference of both the excitation and emission light in the $\mathrm{Al}_{2} \mathrm{O}_{3}$ layer, the fluorophore should be placed close to the surface of the $\mathrm{Al}_{2} \mathrm{O}_{3}$ layer in order to obtain the highest magnitude of the fluorescence enhancement. However, since the size of $E$. coli was larger than the $\mathrm{Al}_{2} \mathrm{O}_{3}$ layer thickness, the maximum fluorescence enhancement was low. It is therefore considered that the fluorescence enhancement obtained in this study was not due to interference, but mainly due to light reflection at the Ag layer.

\section{Confirmation of SOS response-induced GFP expression}

SOS response-induced GFP expression was confirmed using MMC and MNNG as typical mutagens. The E. coli strain transformed with pQBIrecA-GFP was incubated in the LB medium along with varying the concentrations of MMC and MNNG; the fluorescence was measured using a microplate reader. Figures 3(a) and 3(b) show the MMC and MNNG-induced fluorescence intensities of GFP, respectively, as a function of the incubation time. For both mutagens, the fluorescence intensities increased with the incubation time. Moreover, the fluorescence intensities were dependent on the MMC and MNNG concentrations.

If GFP induction is due to the SOS response, the amount of the expressed GFP is dependent on the mutagen concentration, and hence the fluorescence intensity is dependent on the mutagen concentration. On the contrary, if the density of the cell that expresses GFP increases with the mutagen concentration, the fluorescence intensity is also dependent on the mutagen concentration.

To investigate a cause for the increasing fluorescence intensity that is dependent on the mutagen concentration, we measured

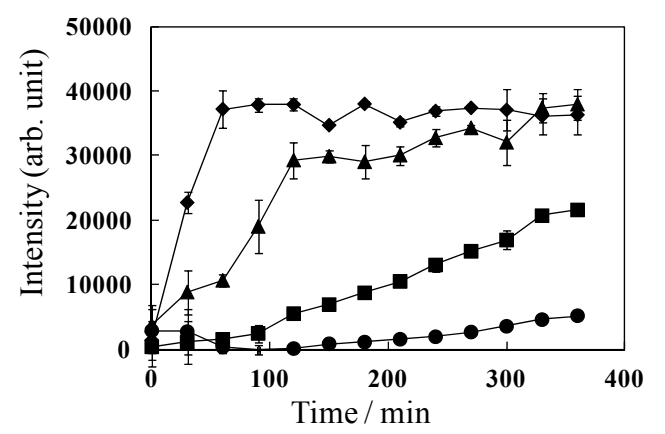

(a)

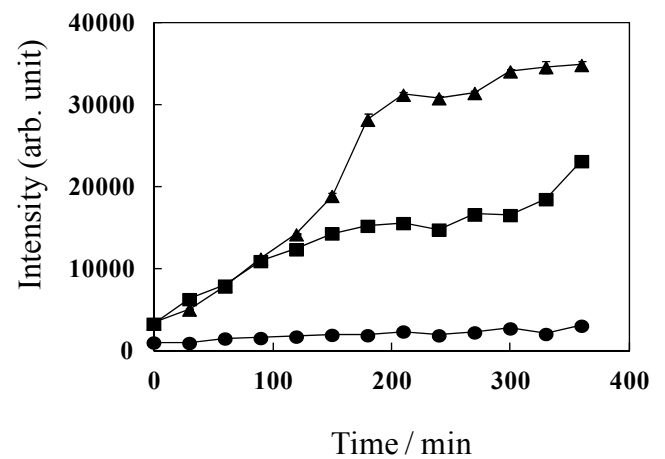

(b)

Fig. 3 Fluorescence intensity of the SOS response-induced GFP change with the incubation time. The $E$. coli strain transformed with pQBIrecA-GFP plasmid was incubated in the LB media in the presence of mutagens, and the fluorescence from the GFP was measured with a microplate reader; $n=5$. (a) MMC: $1000(\diamond), 100(\mathbf{\Delta}), 10(\boldsymbol{\square}), 1(\bullet)$ ng/ml, (b) MNNG: $10(\mathbf{\Delta}), 1(\mathbf{\square}), 0.1(\bullet) \mu \mathrm{g} / \mathrm{ml}$.

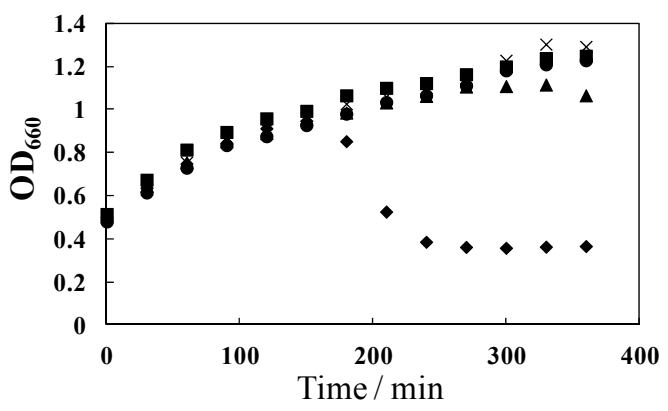

(a)

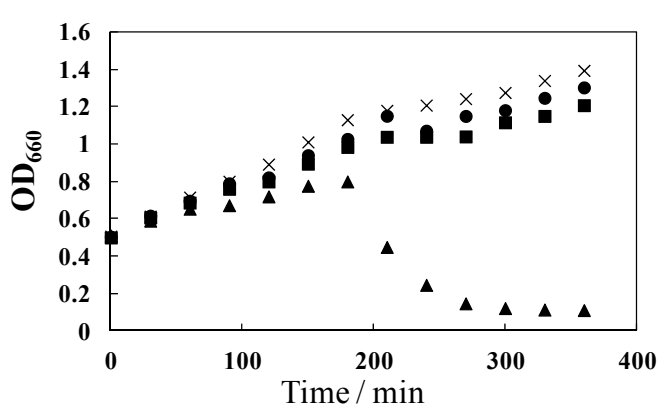

(b)

Fig. 4 Cell density at $\mathrm{OD}_{660}$ change with the incubation time. The cell density of the E. coli strain incubated in LB media in the presence of the mutagens was measured as optical densities at $660 \mathrm{~nm}$. (a)

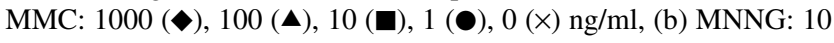
$(\mathbf{\Delta}), 1(\mathbf{\square}), 0.1(\bullet), 0(\times) \mu \mathrm{g} / \mathrm{ml}$. 


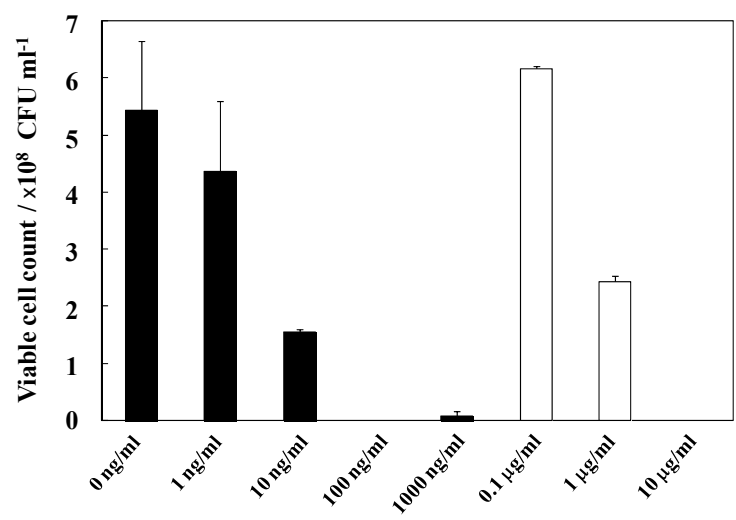

Fig. 5 Viable cell count at $150 \mathrm{~min}$ of the incubation time with MMC ( $\square$ ) and MNNG ( $\square$ ). The cell was incubated with the mutagen for $150 \mathrm{~min}$, and the viable cell count was measured by the plate count. The viable cell counts for 100 and $1000 \mathrm{ng} / \mathrm{ml}$ of MMC, and $10 \mu \mathrm{g} / \mathrm{ml}$ of MNNG were lower than $1 \times 10^{6} ; n=3$.

the changes in the cell density of $E$. coli incubated in the LB medium in the presence of MMC and MNNG. Figures 4(a) and 4(b) show the relationships between the cell density at $\mathrm{OD}_{660}$ and the incubation times of MMC and MNNG, respectively. The cell density increased with the incubation time, and was approximately the same for any concentration of MMC and MNNG within $150 \mathrm{~min}$. This implies that the increase of the fluorescence intensity, depending on the mutagen concentration, can be attributed to the SOS response-induced GFP expression. On the other hand, the decrease of the cell density, probably due to bacteriolysis, was found upon incubation with $1000 \mathrm{ng} / \mathrm{ml}$ $\mathrm{MMC}$ and $10 \mu \mathrm{g} / \mathrm{ml} \mathrm{MNNG}$ for more than $150 \mathrm{~min}$. From this observation, a 150-min incubation time was optimal for evaluating the MMC and MNNG-induced fluorescence of GFP.

The cell density was found to be approximately the same for any concentration within $150 \mathrm{~min}$. However, the cell density involves cells died by mutagens. To assess a viable cell count at 150 min of incubation time, the viable cell count was measured by the plate count (Fig. 5). The viable cell count was found to decrease with increasing the mutagen concentration. Especially for 100 and $1000 \mathrm{ng} / \mathrm{ml}$ of MMC, and $10 \mu \mathrm{g} / \mathrm{ml}$ of MNNG, the viable cell count was lower than $1 \times 10^{6}$. These observations imply that the mutagen induces cell death, even if the incubation time is within 150 min.

Based on these results, the following conclusions can be made: 1) the transformed cells showed SOS response-induced GFP expression; 2) the cell density was approximately the same with concentrations of up to $1000 \mathrm{ng} / \mathrm{ml} \mathrm{MMC} \mathrm{and} 10 \mathrm{mg} / \mathrm{ml}$ MNNG within $150 \mathrm{~min}$ of incubation; and 3) the optimal incubation time used to evaluate the response to $\mathrm{MMC}$ and MNNG was $150 \mathrm{~min}$.

In a further experiment, we only used MMC as the model mutagen, because as a lower concentration of MMC compared with the MNNG-induced SOS response.

\section{MMC detection using E. coli immobilized on a multi-layered} substrate

To detect the SOS response-induced GFP using a multi-layered substrate, the transformed E. coli strain was incubated in the presence of MMC for $150 \mathrm{~min}$. After incubation, the strain was immobilized on a multi-layered substrate. For a comparison, the strain was immobilized on a bare glass substrate using the same immobilization protocol. The calibration using MMC in

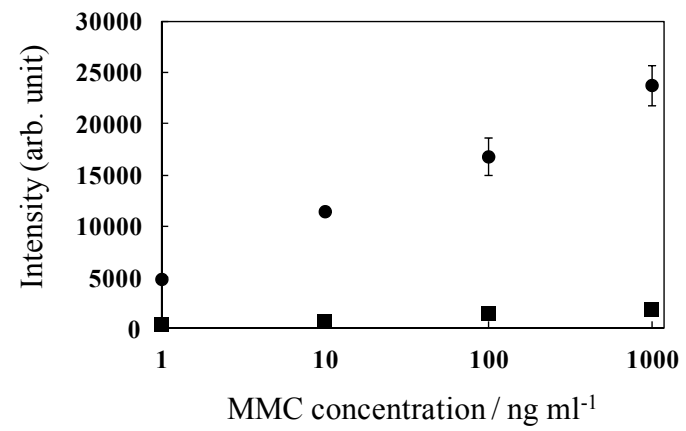

Fig. 6 Calibrations for the MMC concentration obtained with a multi-layered substrate $(\boldsymbol{)})$ and a bare glass substrate $(\boldsymbol{\square})$. The E. coli strain transformed with pQBIrecA-GFP was incubated in the presence of MMC, and immobilized on the multi-layered substrate with a 50-nm thick $\mathrm{Al}_{2} \mathrm{O}_{3}$ layer and the bare glass substrate. The multi-layered substrate and the bare glass substrate were treated with APTS, and used to immobilize the strain with glutaraldehyde. The fluorescence intensities were measured by a fluorescence imager; $n=5$.

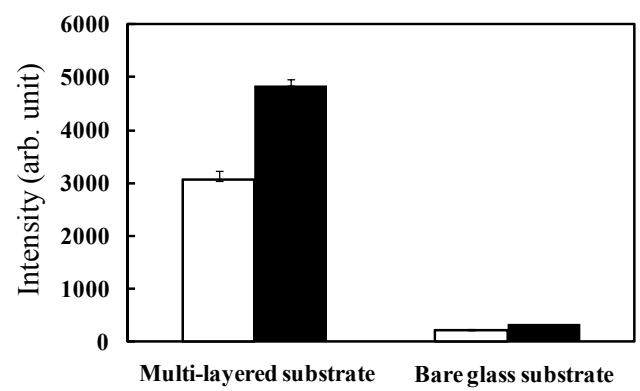

Fig. 7 Comparison of the fluorescence intensity of the SOS response-induced GFP for $0 \mathrm{ng} / \mathrm{ml}(\square)$ and $1 \mathrm{ng} / \mathrm{ml}(\boldsymbol{\square})$ of MMC concentration. The strain that express the GFP was immobilized on a multi-layered substrate and a bare glass slide. The difference between the signal of $1 \mathrm{ng} / \mathrm{ml}$ and that of $0 \mathrm{ng} / \mathrm{ml}$ was found to be enlarged by the multi-layered substrate.

the concentration range from 1 to $1000 \mathrm{ng} / \mathrm{ml}$ is shown in Fig. 6 .

The fluorescence intensity using the multi-layered substrate was higher than that using the bare glass substrate. To calculate the enhancement factor, the fluorescence intensity using the multi-layered substrate was divided by that using the glass substrate for each MMC concentration. On average, a 14-fold enhancement was observed. However, considering the above discussion, a 25 -fold enhancement should have been achieved. We were unable to find a reason for these contradictory results; however, they could have been due to the amount of GFP that was expressed. The amount of SOS response-induced GFP expressed should be less than that induced by the $\mathrm{T} 7$ promoter. Since the enhancement factor depends on the amount of the fluorophore, the enhancement factor obtained by the SOS response is smaller than that by the $\mathrm{T} 7$ promoter.

To estimate the lower detection limit for the MMC concentration, the fluorescence intensities using the multi-layered substrate and the bare glass substrate for MMC concentration of 0 and $1 \mathrm{ng} / \mathrm{ml}$ were compared (Fig. 7). When the detection limit is defined as a signal beyond a blank plus a $3 \times$ standard deviation of the blank, $1 \mathrm{ng} / \mathrm{ml}$ MMC could not be detected using the glass substrate, but was detectable using the multi-layered substrate. Therefore, we concluded that the lower 
detection limit could be improved by using the multi-layered substrate. We consider that this improvement was due to an enlargement of the difference between the signal of $1 \mathrm{ng} / \mathrm{ml}$ and that of $0 \mathrm{ng} / \mathrm{ml}$ by the multi-layered substrate.

\section{Conclusions}

In this study, we described mutagen detection using genetically modified E. coli immobilized on a multi-layered substrate. We found that a maximum 25 -fold fluorescence enhancement of GFP, which was not induced by an SOS response, could be obtained with a 50-nm thick $\mathrm{Al}_{2} \mathrm{O}_{3}$ layer. However, it was thought that this enhancement was not attributed to optical interference, but to simple reflection at the $\mathrm{Ag}$ layer. This was due to the large size of $E$. coli compared with the $\mathrm{Al}_{2} \mathrm{O}_{3}$ layer thickness in the multi-layered substrate.

We constructed genetically modified E. coli capable of expressing mutagen-induced GFP. The optimal incubation time for MMC and MNNG-induced GFP was estimated to be 150 min. The cell density was the same for any concentration of MMC and MNNG within this time, but the viable cell count deceased with increasing the mutagen concentration at this time.

To detect MMC, transformed E. coli cells that expressed MMC-induced GFP were immobilized on the multi-layered substrate. The average fluorescence enhancement was 14-fold for the MMC concentration in the range of 1 to $1000 \mathrm{ng} / \mathrm{ml}$. The lower detection limit for MMC using the multi-layered substrate was estimated to be $1 \mathrm{ng} / \mathrm{ml}$, which was an improvement compared with that using a bare glass substrate. We consider that this improvement was due to an enlargement of the difference between the signal of $1 \mathrm{ng} / \mathrm{ml} \mathrm{MMC}$ and that of $0 \mathrm{ng} / \mathrm{ml} \mathrm{MMC}$ by the multi-layered substrate.

\section{References}

1. B. N. Ames, Science, 1979, 204, 587.

2. P. Quillardet, O. Huisman, R. D'ari, and M. Hofung, Proc.
Natl. Acad. Sci. U. S. A., 1982, 79, 5971.

3. S. J. Sørensen, M. Burmølle, and L. H. Hansen, Curr. Opin. Biotechnol., 2006, 17, 11.

4. L. R. Ptitsyn, G. Horneck, O. Komova, S. Kozubek, E. A. Krasavin, M. Bonev, and P. Rettberg, Appl. Environ. Microbiol., 1997, 63, 4377.

5. M. Shirakawa, T. Tsurimoto, and K. Matsubara, Gene, 1984, 28, 127.

6. R. Arai, Y. Makita, Y. Oda, and T. Nagamune, J. Biosci. Bioeng., 2001, 92, 301.

7. M. Kostrzynska, K. T. Leung, H. Lee, and J. T. Trevors, J. Microbiol. Methods, 2002, 48, 43.

8. A. Norman, L. Hestbjerg, and S. Sørensen, Mutat. Res., 2006, 603, 164.

9. N. L. Craig and J. W. Roberts, Nature, 1980, 283, 26.

10. G. C. Walker, Microbiol. Rev., 1984, 48, 60.

11. C. Janion, Int. J. Biol. Sci., 2008, 4, 338.

12. K. C. Giese, C. B. Michalowski, and J. W. Little, J. Mol. Biol., 2008, 377, 148

13. A. Vollmer, S. Belkin, D. Smulski, T. Van Dyk, and R. LaRossa, Appl. Environ. Microbiol., 1997, 63, 2566.

14. A. Norman, L. Hestbjerg, and S. Sørensen, Appl. Environ. Microbiol., 2005, 71, 2338.

15. W. R. Holland and D. G. Hall, Opt. Lett., 1985, 10, 414.

16. K. G. Sullivan, O. King, C. Sigg, and D. G. Hall, Appl. Opt., 1994, 33, 2447.

17. T. Akimoto, M. Yasuda, and I. Karube, Appl. Opt., 2008, 47(21), 3789.

18. T. Akimoto and M. Yasuda, Appl. Opt., 2010, 49(1), 80.

19. E. Le Moal, E. Fort, S. Lévêsque-Fort, F. P. Cordelières, M.-P. Fontaine-Aupart, and C. Ricolleau, Biophys. J., 2007, $92,2150$.

20. E. Le Moal, E. Fort, S. Léve Que-Fort, A. Janin, H. Murata, F. P. Cordelières, M. P. Fontaine-Aupart, and C. Ricolleau, J. Biomed. Opt., 2007, 12, 024036.

21. O. G. Berg, Nucleic Acids Res., 1988, 16, 5089.

22. L. K. Lewis, G. R. Harlow, L. A. Gregg-Jolly, and D. W. Mount, J. Mol. Biol., 1994, 241, 507. 